\title{
Using the IBM-compatible microcomputer's serial port as an input-output interface
}

\author{
C. CRAIG MORRIS \\ Baylor University, Waco, Texas
}

\begin{abstract}
A general purpose input-output interface is an essential tool in many common research situations. This article describes a simple way to set up the IBM-compatible microcomputer's RS-232 serial port as an interface with up to four inputs and two outputs. First, aspects of the serial port relevant to its use as an input-output interface are discussed. Next, port access commands needed to control output lines and monitor input lines in the appropriate way are described, and examples in the Turbo Pascal 5.0 language are provided. Finally, a schematic diagram and a discussion of a simple circuit to implement the interface are provided.
\end{abstract}

Much current research in cognitive psychology involves the use of IBM-compatible microcomputers to present stimuli and observe aspects of responding such as accuracy and latency. Although numerous articles have described ways to program these microcomputers for accurate timing in such studies (Bovens \& Brysbaert, 1990; Brysbaert, 1990; Brysbaert, Bovens, d'Ydewalle, \& Van Calster, 1989; Bührer, Sparrer, \& Weitkunat, 1987; Crosbie, 1989; Dlhopolsky, 1989; Emerson, 1988; Graves \& Bradley, 1987, 1988; Heathcote, 1988), less attention has been paid to the equally important issue of how to interface them to external devices. One problem is that the accuracy of timing also depends on the input device used to measure responding, including the keyboard. For example, Graves and Bradley (1987) found a mean reaction time detection delay of $18 \mathrm{msec}$ for the IBM PC keyboard and $36 \mathrm{msec}$ for a clone keyboard. And Segalowitz and Graves (1990) reported a mean delay of $10-15 \mathrm{msec}$ and a random error of $\pm 5-7.5 \mathrm{msec}$ for IBM microcomputer keyboards, depending on the key and type of machine. Another problem is that the keyboard is not a useful response device in many situations. In word recognition studies, for example, a common response is word naming latency, usually measured by a voice-activated switch connected to a computer (Seidenberg, Waters, Sanders, \& Langer, 1984). One way to interface external switches to an IBM-compatible microcomputer is to use the game port, which on the XT can be connected to up to four switches and which yields better timing accuracy than the keyboard does (Segalowitz \& Graves, 1990). However, some popular IBM-compatible microcomputers do not implement the game port (e.g., the Zenith 181 laptop). A third problem is that studies often require the computer to both monitor external switches and control external devices, such as projectors and audio or video recorders. Thus, a general purpose

Correspondence should be addressed to C. C. Morris, Psychology Department, Middle Tennessee State University, Murfreesboro, TN 37132. input-output interface is an essential tool in many common research situations.

Some elaborate interfaces for microcomputers have been described in the literature (Arnold \& Hastings, 1984; Krausman, 1978; Lavond \& Steinmetz, 1989; Stoddard \& Loftus, 1988). These interfaces permit many inputs and outputs, but building and trouble-shooting their complex electronic circuits may require more skill in electronics than most psychologists possess. Also, it is not prudent to build an interface more complex than is required for the research. Emerson (1988) noted that the RS-232 serial port can be used to set up a simple input-output interface for IBM-compatible computers. However, because his focus was on stimulus presentation and timing in reaction time experiments, his discussion of a serial port interface was brief and preliminary. He implied that only three inputs can be monitored when one uses the serial port's binary input (modem status) register, although the maximum is four, and he described using a pair of $9-\mathrm{V}$ batteries to power the interface, although it is preferable to use an AC-converter dual-polarity power supply. Finally, he did not point out that the current on each output pin in the serial port is too weak to drive a relay, which is needed to control many external devices, although that current will drive a switching transistor connecting the relay to an adequate current from an external power supply.

The purpose of the present article is to provide a tutorial describing how to use the RS-232 serial port as an input-output interface capable of monitoring four inputs and controlling two outputs. The minimal hardware and software demands of this interface make it readily accessible to a broad population of psychological researchers. The two required port access commands can be translated from the Turbo Pascal 5.0 (Borland, 1989) commands provided here into any computer language that provides port access commands. In the following, I first discuss aspects of the RS-232 serial port relevant to its use as an input-output interface. Next, I describe how to use computer commands to monitor input lines and control output lines at the serial port in the appropriate way. Finally, 
I present a schematic diagram of a simple circuit designed to set up the serial port to function as an input-output interface.

The RS-232 serial port is designed to connect the microcomputer to a modem or to other data communication equipment (Campbell, 1984; Radio Shack, 1984). There are either 25 or 9 pins in the RS-232 connector at the back of the IBM-compatible computer. The 25-pin connector typically appears on 8088-type machines, whereas the 9-pin connector typically appears on 80286 and later machines. For clarity of exposition, I will discuss line numbers for the 25-pin connector and place the corresponding line numbers for the 9-pin connector in parentheses. The pins may be numbered on the connector, or on a cable connector, but a magnifying glass may be needed to read them. The pins in the 25-(9-)pin connector lie in two parallel rows of $13(5)$ and $12(4)$ pins each. Facing the back of the computer, with the wide row above the narrow row, the pins in the upper row are numbered 1 (1) to 13 (5), left to right, and the pins in the lower row are numbered 14 (6) to $25(9)$, left to right. Table 1 lists each pin, its signal function, its common abbreviation, and its status as an input or output line relative to the computer.

Of the 25 (9) pins in the connector, 7 are needed for our purposes. These are the modem control output pins 4 (7) and 20 (4) (denoted RTS and DTR, respectively), the modem control input pins $5(8), 6(6), 8(1)$, and 22 (9) (denoted CTS, DSR, DCD, and RI, respectively), and the signal ground pin 7 (5) (denoted SG). These are lines by which the computer and modem communicate their readiness to interact with each other. Whereas the two output lines are designed to send the signals Ready To Send (RTS) and Data Terminal Ready (DTR) to the modem, the four input lines are designed to receive the signals Clear To Send (CTS), Data Set Ready (DSR), Data Carrier Detect (DCD), and Ring Indicator (RI), from the modem. The binary logical status of each line is signaled electrically by the polarity of the voltage on that line relative to signal ground on line 7 (5). For these six modem control lines, a positive voltage (between +3 and $+15 \mathrm{~V}$ ) represents the logical high (on) state, whereas a negative voltage (between -3 and $-15 \mathrm{~V}$ ) represents the logical low (off) state (Campbell, 1984). (For some other lines in the RS-232 port, called data lines, the opposite polarities represent logical low and high; see Radio Shack, 1984, p. 4.17.) Some IBM-compatible microcomputers may contain modifications of the Intel 8250 UART (universal asynchronous receiver transmitter) chip supporting serial communications that have changed or deleted some of the lines and functions listed in Table 1. However, lines and functions corresponding to pins in the 9-pin connector are not likely to have been modified.

The input lines CTS, DSR, DCD, and RI are high (low) if connected to a voltage source that is between +3 and $+15 \mathrm{~V}(-3$ and $-15 \mathrm{~V})$ relative to signal ground. To assess the current logical status of the input lines, it is necessary to read the byte in the modem status register, located at a certain port address. The output lines RTS and DTR can be set high or low by a computer command

Table 1

Pins in the RS-232 Serial Port Connector

\begin{tabular}{|c|c|c|c|c|}
\hline Pin & & Signal Description & Abbreviation & $\mathrm{I} / \mathrm{O}$ \\
\hline 1 & & Protective (Chassis) Ground & GND & \\
\hline 2 & (3) & Transmitted Data & TD & $\mathbf{O}$ \\
\hline 3 & (2) & Received Data & RD & I \\
\hline 4 & (7) & Request to Send & RTS & $\mathrm{O}$ \\
\hline 5 & (8) & Clear to Send & CTS & I \\
\hline 6 & (6) & Data Set Ready & DSR & I \\
\hline 7 & (5) & Signal Ground/Common Return & SG & \\
\hline 8 & (1) & Received Line Signal Detector & DCD & I \\
\hline 9 & & Reserved & & \\
\hline 10 & & Reserved & & \\
\hline 11 & & Unassigned & & \\
\hline 12 & & Secondary Received Line Signal Detector & & I \\
\hline 13 & & Secondary Clear to Send & & I \\
\hline 14 & & Secondary Transmitted Data & & $\mathrm{O}$ \\
\hline 15 & & Transmitter Signal Element Timing (DCE) & & I \\
\hline 16 & & Secondary Received Data & & I \\
\hline 17 & & Receiver Signal Element Timing & & I \\
\hline 18 & & Unassigned & & \\
\hline 19 & & Secondary Request to Send & & $\mathrm{O}$ \\
\hline 20 & (4) & Data Terminal Ready & DTR & $\mathbf{O}$ \\
\hline 21 & & Signal Quality Detector & SQ & I \\
\hline 22 & (9) & Ring Indicator & RI & I \\
\hline 23 & & Data Signal Rate Selector & & \\
\hline 24 & & Transmitter Signal Element Timing (DTE) & & O \\
\hline 25 & & Unassigned & & \\
\hline
\end{tabular}

Note-Pins on the 9-pin connector appear in parentheses. Input (I) and output (O) lines are defined as inputs to, or outputs from, the computer. (From "Understanding Data Communications" by Radio Shack, 1986, 4-10, p. 4-16. Copyright 1986 by Radio Shack. Adapted by permission.) 
Table 2

Bit Assignments in the Modem Status and Modem Control Registers

\begin{tabular}{cll}
\hline Bit & \multicolumn{1}{c}{ Modem Status Register } & \multicolumn{1}{c}{ Modem Control Register } \\
\hline 0 & Change in CTS Logical Status & DTR Logical Status \\
1 & Change in DSR & RTS Logical Status \\
2 & Change in RI & Auxiliary User Designated Output 1 \\
3 & Change in DCD & Auxiliary User Designated Output 2 \\
4 & CTS Logical Status & Loop UART Output Back As Input \\
5 & DSR Logical Status & (Always 0) \\
6 & RI Logical Status & (Always 0) \\
7 & DCD Logical Status & (Always 0) \\
\hline
\end{tabular}

Note-Bits 2-7 in the modem control register should remain set to zero. (From Programmer's Problem Solver for the IBM PC, XT and AT (pp. 378-379) by R. Jourdain, 1986, New York: Brady/A Division of Simon and Schuster. Adapted by permission.)

and will remain set unless another command changes that logical status. To set either output line high or low, it is necessary to place the appropriate byte in the modem control register, located at a different port address. To understand how the logical status of the input and output lines is represented by the bytes in the modem status and modem control registers, recall that a byte consists of 8 binary digits, or bits, each with the value 0 or 1 . The bits are numbered from 0 to 7 , right to left, with each successive number corresponding to that power of 2 . The byte in either register indicates the logical status of all the bits in that register. Each of the four input lines is assigned to a unique bit in the modem status register, with the logical status of each line indicated by the value of the corresponding bit-that is, $0=$ low, $1=$ high. Similarly, each of the two output lines is assigned to a unique bit in the modem control register, with the logical status of each line determined by the value of the corresponding bit. DOS, the most common IBM-compatible operating system, supports two separate communications channels, COM1 and COM2. The address of the modem control register is the COM1 base address plus 4 -that is, $3 F 8 \mathrm{~h}+4$ $=3 \mathrm{FCh}-$ while the address of the modem status register is the COM 1 base address plus 6 - that is, $3 \mathrm{~F} 8 \mathrm{~h}+6=3 \mathrm{FEh}$ (on PCjr computers, the base address is $2 \mathrm{~F} 8 \mathrm{~h}$ ). These addresses are hexadecimal numbers. See Norton (1986) for a discussion of computer arithmetic, port and memory addressing, and other useful information. Table 2 indicates the assignment of lines to bits in the modem status and modem control registers (Jourdain, 1986, pp. 378-379).

The logical status of the output lines DTR and RTS is determined by the binary value of bits 0 and 1 , respectively, in the modem control register. Thus, putting the number $0 \mathrm{~b}=0$ in that register will set both DTR and RTS low, putting $1 \mathrm{~b}=1$ in will set DTR high and RTS low, putting $10 \mathrm{~b}=2$ in will set DTR low and RTS high, and putting $11 \mathrm{~b}=3$ in will set both DTR and RTS high. Bits 2-7 in the modem control register should always remain set to zero, so $0,1,2$, and 3 are the only values that should ever be placed in this register. In Turbo Pascal 5.0, the operation of placing a byte in the modem control register is carried out by the following port access command, where the variable B is of type BYTE and set to the desired value to be placed in the register:

$$
\text { PORT[\$3FC] := B. }
$$

Of course, one can replace $B$ with the desired value itself $(0,1,2$, or 3$)$ in this expression.

The current logical status of the input lines CTS, DSR, $R I$, and DCD is represented by the value of bits $4,5,6$, and 7 , respectively, in the modem status register. Bits 0 , 1,2 , and 3 in this register indicate whether the logical status of an input line has changed since the last time the register was read. To observe the logical status of the four input lines at a given moment, read the byte in the modem status register and set its bits $0-3$ to zero by taking the logical AND of the result with $11110000 \mathrm{~b}=240$-for example, $10001000 \mathrm{~b}$ AND $11110000 \mathrm{~b}=10000000 \mathrm{~b}=$ 128. In Turbo Pascal 5.0, the operation of reading the modem status register and taking the AND of the result with 240 is carried out by the following command, where the variable $B$ is of type BYTE:

$$
\text { B := PORT[\$3FE] AND } 240 .
$$

If only one of the four input lines is high, the result is 16 (CTS), 32 (DSR), 64 (RD), or 128 (DCD), and the high bit number equals RND(LN(B)/LN(2)). If two or more lines are high, the result identifies the combination; for example, $10010000 \mathrm{~b}=16+128=144$ (CTS and DCD).

The circuit diagrammed in Figure 1 provides a serial port input-output interface capable of monitoring four input lines and controlling two output lines. ${ }^{1}$ The components of the circuit are an RS-232 serial port connector, a dual-polarity power supply (e.g., Elpac Power Systems, 87F2913), two transistors (e.g., Phillips, ECG123AP), two diodes (e.g., Phillips, ECG109), two 5-6 V reed relays with diode protection (e.g., Phillips, RLYF71A05C), two $680-\Omega$ resistors, four $2.2-\mathrm{k} \Omega$ resistors, and four input switches. If only an input or output interface is needed, only that part of the circuit (bottom or top in diagram) need be built. If fewer than four inputs or two outputs are needed, the components and circuitry for the other inputs and outputs can be omitted. The only connections common to both input and output operations are those con- 


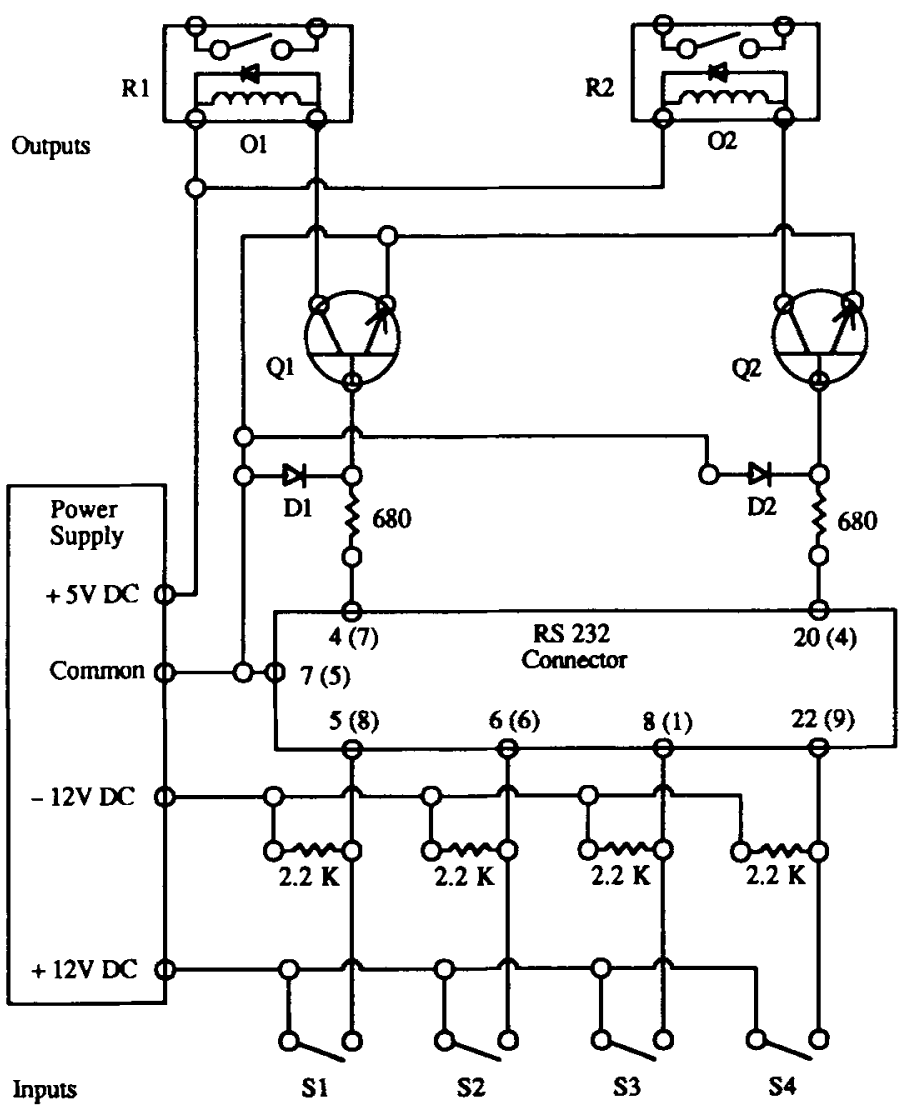

Figure 1. Schematic dingram of a circult providing a serial port input-output interface.

necting the ground terminal of the power supply to the signal ground terminal, line 7 (5), of the RS-232 connector. Once the desired circuit is assembled, it should be tested by verifying that the voltage at the RS-232 connector across signal ground and each input or output line falls within the limits of $\pm 3-15 \mathrm{~V}$, with the proper sign, both when all switches and relays are open and when switches and relays are being operated in each possible combination (e.g., verify that the voltage across pins 5 (8) and $7(5)$ is between -3 and $-15 \mathrm{~V}$ when switch $S 1$ is open with switches S2, S3, and S4 all closed). During the debugging stage, it is helpful first to plug an RS-232 line tester into the computer's RS-232 connector and then to plug the connector wired to the interface into the other side of the line tester. The line tester has light-emitting diodes that signal the logical status of the modem status and modem control lines, making it easy to see whether the lines are being activated and disactivated properly. The line tester should be removed during voltage testing to ensure that voltages remain within prescribed limits.

The general functioning of the circuit in Figure 1 is as follows. On the input side of the interface, whenever a switch is open, a negative voltage from the power supply is applied through the $2.2-\mathrm{k} \Omega$ resistor to the correspond- ing input line on the RS-232 connector. The voltage across that input line and signal ground will be between -3 and $-15 \mathrm{~V}$, so the line will be logic low. When the switch is closed, however, a positive voltage from the power supply is applied to the input line; the voltage across that input line and signal ground will be between +3 and $+15 \mathrm{~V}$, so the line will be logic high. On the output side of the interface, whenever an output line is high, a positive voltage is applied through a $680-\Omega$ resistor to the base of an NPN transistor (Q1 or Q2), saturating the transistor and completing the circuit through its other two connections, thereby activating the relay by completing a circuit connecting the relay to $+5 \mathrm{~V}$ and ground at the power supply. Whenever the output line is low, however, a small negative voltage is applied to the base of the transistor, and the circuit through its other two connections remains closed, preventing activation of the relay. Diodes D1 and D2 ensure that the negative voltages from the (low) output pins do not exceed the reverse bias ratings of the transistors, which could damage them or cause them to activate the relays, by allowing current from the negative output pins to flow (through the 680- $\mathrm{n}$ resistor) to ground. Note that the outputs 01 and 02 supply $+5 \mathrm{~V}$ whenever the output lines RTS, pin 4 (7), and DTR, pin 20 (4), are 
high. If desired, the relays connected to these outputs can be replaced (or supplemented) with other devices, such as light-emitting diodes. Dual-pole dual-throw switches can be installed at connections $\mathrm{O} 1$ and $\mathrm{O} 2$ to permit switching between the indicated relays and other devices. It is a good idea to solder receptacles for the relays to the circuit board, so that the relays can easily be inserted and removed-to permit use of relays with different contact voltage and current ratings, for example, or to permit use of the outputs $\mathrm{O} 1$ and $\mathrm{O} 2$ for a different current demanding task.

Finally, if only one input line is required, there is an even simpler way to implement a serial port interface requiring only one resistor, one switch, and no external power supply. Connect one output line (DTR) directly to the input switch, and connect the other output line (RTS) through a resistor to the other side of the switch. Then set DTR high and RTS low with the command Port[\$3FC] $:=1$. Whenever the switch is open (closed), the input line will be low (high). The resistor size (about $2.2-\mathrm{k} \Omega$ ) must be chosen to ensure that voltages fall within the required limits of $\pm 3-15 \mathrm{~V}$, with the proper sign, when the switch is open and closed. If enough voltage is being supplied to the output pins on a particular machine, it may be possible to implement more than one input line by using the DTR and RTS output lines to supply positive and negative voltages, just as multiple input lines appear in Figure 1 . In any case, the test recommended above should be used to verify that voltages are within prescribed limits under all circumstances.

\section{REFERENCES}

Arnold, N. D., Hastings, L. (1984). A bit-addressable I/O interface for microcomputers. Behavior Research Methods, Instruments, \& Computers, 16, 135-136.

Borland InTERNATIONAL, InC. (1989). Turbo Pascal reference guide (Version 5.0). Scotts Valley, CA: Author.

Bovens, N., BRYSBAert, M. (1990). IBM PC/XT/AT and PS/2 Turto Pascal timing with extended resolution. Behavior Research Methods, Instruments, \& Computers, 22, 332-334.

BrysBaERT, M. (1990). A warning about millisecond timing in Turbo Pascal. Behavior Research Methods, Instruments, \& Computers, 22, 344-345.

Brysbaert, M., Bovens, N., D'Ydewalle, G., \& Van Calster, J. (1989). Turbo Pascal timing routines for the IBM microcomputer family. Behavior Research Methods, Instruments, \& Computers, 21 , 73-83.

Bührer, M., Sparrer, B., \& Weitkunat, R. (1987). Interval timing routines for the IBM PC/XT/AT microcomputer family. Behavior Research Methods, Instruments, \& Computers, 19, 327-334.

Camprell, J. (1984). The RS-232 solution. Alameda, CA: Sybex.

Crosbie, J. (1989). A simple Turbo Pascal 4.0 program for millisecond timing on the IBM PC/XT/AT. Behavior Research Methods, Instruments, \& Computers, 21, 408-413.

DLhopolsKY, J. G. (1989). Synchronizing stimulus displays with millisecond timer software for the IBM PC. Behavior Research Methods, Instruments, \& Computers, 21, 441-446.

EMERSON, P. L. (1988). Using serial interfaces and the C language for real-time experiments. Behavior Research Methods, Instruments, \& Computers, 20, 330-336.

Graves, R., Bradley, R. (1987). Millisecond interval timer and auditory reaction time programs for the IBM PC. Behavior Research Methods, Instruments, \& Computers, 19, 30-35.

Graves, R., Bradley, R. (1988). More on millisecond timing and tachistoscope applications for the IBM PC. Behavior Research Methods, Instruments, \& Computers, 20, 408-412.

HeAtHCote, A. (1988). Screen control and timing routines for the IBM microcomputer family using a high-level language. Behavior Research Methods, Instruments, \& Computers, 20, 289-297.

JourdaIn, R. (1986). Programmer's problem solver for the IBM PC, $X T \& A T$. New York: Prentice-Hall.

KraUSmaN, D. T. (1978). Interfacing the microcomputer: A componentlevel approach. Behavior Research Methods, Instruments, \& Computers, 10, 519-521.

Lavond, D. G., Steinmetz, J. E. (1989). An inexpensive interface for the IBM PC/XT and compatibles. Behavior Research Methods, Instruments, \& Computers, 21, 435-440.

Norton, P. (1986). Peter Norton's assembly language book for the IBM PC. New York: Prentice-Hall.

RADIO SHACK (1984). Understanding data communications. Indianapolis: Howard W. Sams.

Segalowitz, S. J., Graves, R. E. (1990). Suitability of the IBM $\mathrm{XT}, \mathrm{AT}$, and PS/2 keyboard, mouse, and game port as response devices in reaction time paradigms. Behavior Research Methods, Instruments, \& Computers, 22, 283-289.

Seidenberg, M. S., Waters, G. S., Sanders, M., * Langer, P. (1984). Pre- and postlexical loci of contextual effects on word recognition. Memory \& Cognition, 12, 315-328.

Stoddard, P. K., \& LofTus, G. R. (1988). An IBM XT-compatible, computer-based, slide-projector laboratory. Behovior Research Methods. Instruments, \& Computers, 20, 541-551.

\section{NOTE}

1. I thank Mark Roessler for suggesting this circuit design.

(Manuscript received May 8, 1991;

revision accepted for publication November 13, 1991.) 